\title{
Design Improvementof Steering Upright by Investigating Static and Dynamic Analysis
}

\author{
Ipsita Swain*, Subim N Khan ${ }^{\#}$ \\ * P.G Scholar, Dept. of Mechanical Engineering, Rajarshi Shahu College of Engineering, Pune \\ \#Associate Professor, Dept. of Mechanical Engineering, Rajarshi Shahu College of Engineering, Pune
}

(Received 22 June 2020, accepted 03 November 2020)

https://doi.org/10.36224/ijes.130402

\begin{abstract}
A steering upright is one of the vital components in vehicle dynamics which links all suspension components between the wheel and therefore the vehicle. Upright provides the linkage between the upper \& lower ball joints. The upright connects components as an example, control arms, steering arms, suspension springs, shock absorber, brake disc, wheels \& tire. Steering upright is a part of mechanical system which contains the wheel hub and assembles to the suspension components. It's the pivot point of the steering and mechanical system, which allows the front wheels to show \& more. Agenda of this project is design optimization of steering upright to achieve weight reduction along with required strength and stiffness. Optimized design of upright is necessary with correct material selection further as valid finite element analysis. Optimization is accomplished considering static stress, strain and total deformation analysis with acceptable material selection. Modal and Fourier analysis is to be performed to see natural frequencies and deformation. The experimental testing is going to be performed on UTM. By making the comparative analysis, result and conclusion is drawn.
\end{abstract}

Keywords: Steering Upright, Stiffness, ANSYS19, UTM

\section{Introduction}

In the automobile sector robust and comfortless of the system is that the most significant thing. The auto should maintain the comfort not only on the standard roads but also on off roads too. For that purpose a high quality steering mechanism is most significant thing, because it won't just absorb the vibrations but also supports the full system. Steering upright is the part which accommodates the wheel hub or spindle, and links to the suspension and steering components. It's variously called a steering knuckle, spindle, upright or hub also. The knuckle is attached to the upper control arm at the very best and so the lower control arm at the lowest.

In a non-drive suspension, as shown within the primary photo, the knuckle usually features a spindle onto which the drum or brake rotor attaches. in an exceedingly drive suspension, the knuckle has no spindle, but rather features a hub into which is affixed the bearings and shaft of the drive mechanism. the top of the drive mechanism would then have the specified mounting studs for the wheel/tire and/or brake assembly. During this automotive and competitive era the need for light-weight weight structural materials is increasing as there's a additional think about fuel consumption reduction and improvement in decreasing the emission. The magnitude of production volumes has historically placed severe necessities on the robustness of method employed within the manufacturing. The manufacturers have strong importance on the value has the demand for the component to reinforce the fabric performance and to deliver these materials at low cost is that the requirement. 


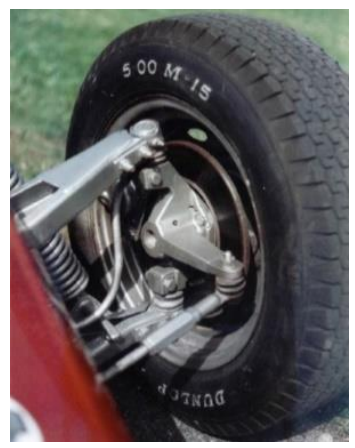

Figure 1: Existing steering mount assembly

The steering knuckle is that the pivot point of the mechanism, which allows the wheels to revolve. Generally on automobile vehicle with conventional suspension systems, the steering knuckle's spindle locates and supports the inner and outer wheel bearings. Upright is also a part of the wheel assembly which holds the hub and allows rotation of the wheel. Forces from the tire's contact patch are conveyed by the upright to the suspension linkages. Hence the steering upright should have that much of strength in such a fashion that it mustn't fail in off road conditions because the terrain is incredibly difficult and its function isn't to only support the auto but also to transfer the steering motion to the wheels coming from mechanism. So there's a high possibility of failure thanks to the sudden stress generation and resonance. Even slight damage of the steering knuckle can affect the correct wheel alignment and disturb the stableness of the vehicle. Therefore this component should be manufactured with high precision and sturdiness.

\section{Literature review}

Mahesh P. Sharma et al. [1] in this paper Steering knuckle is one of the important component which connects to steering, suspension and brake to chassis of auto. It undergoes different loading under various conditions. In this paper static analysis of steering knuckle was done. Knuckle was designed which accommodates twin calliper mountings to improve braking efficiency \& reduce stopping distance of a car. During this investigation, steering knuckle was used as component for study. Main design and functionality of steering knuckle depends on variety of suspension implemented. Additional factors like brake calliper, steering sub-system rod mounting also effects knuckle design. To result maximum stress and deformation of steering knuckle when different forces like braking force, load transfer during acceleration and braking etc. are applied thereon static analysis is performed. Structural optimization tools like topology and shape optimization alongside manufacturing simulation have gotten attractive tools in product design process. These tools also help to reduce development time. Shape optimization gives the optimum fillets and thus the optimum outer dimensions.

V. Sivananth et al. [2] Simulation and analysis of components are executed in automobile plant, so as to cut back the quantity of prototype during experimental validation. During this study, CAD model of steering knuckle was developed using modeling package SOLIDWORKS. While vehicle in a motion, the load generated at tire road contact patch would be transferred to the knuckle and its surrounded parts. The load transfer varies relation to various driving condition such as cornering, acceleration, braking, bump, static pot hole etc. will severely affect its fatigue life. It's been noticed from knuckle manufacturers that material and geometric optimization is that the real need for the automobile industry to reduce weight of the knuckle 
without affecting the performance. Static analysis was performed on three load cases and the results were compared for SG iron and Al alloy.

Gaurav Saxena et al. [3] this paper carries different methodologies adopted by researcher for wheel hub and upright assembly analysis with main goal of study \& optimization on vehicle. This review assists researchers working within the sphere of development of structural design and mass reduction of auto by optimization method conducted through FEA software viz. Creo 1.0 and Hyper Works. In Wheel hub and upright assembly may be a very critical a part of the vehicle suspension which allows the steering arm to indicate the front wheels and support the vertical weight of the vehicle. Upright is additionally referred as the knuckle.

Saksham Bhardwaj et al. [4] Steering upright is a part of suspension system which contains the wheel hub, and attaches to the suspension components. It is the pivot point of steering and suspension aggregate, which allows the front wheels to turn. Considering it for double wishbone suspension geometry, lightweight \& low fuel consumption are fundamental requirements for vehicle, especially for racing car. This paper focus on design optimization of steering upright/ knuckle target to induce weight reduction with required strength and stiffness. Our project provides two paths to realize identical goals of weight reduction, one which needs only a few resources and hence the other requires several resources. First approach of intuition shall be used for low budget study by compromising a bit on the burden and increasing the target Factor of safety.

Mahendra L. Shelar et al. [5] In these journal it presents the foremost important issue in vehicle industry is that the existence of differences within the physical properties and manufacturing methodologies. Deterministic approaches are incapable to into account these variability's without leading to oversized structures. The requirement of assessing the robustness of a particular design requires a method supported strength and elegance optimization through probabilistic models of design variables (DOE). Generally, it's identified the steering knuckle which is one amongst the critical components of auto which links suspension, steering system, wheel hub and brake to the chassis. In these journal author have identified the above problem strategy of optimizing the planning employing a strategy supported durability and elegance optimization through probabilistic models of design variables (DOE). When comparing the optimized model with the initial version, $9.19 \%$ weight has been reduced with acceptable stress and deflection change and not crossing the project target limits.

\section{Problem statement}

Steering upright is used for transmission of motion from steering system to the wheels. They're assembled by using the kingpin, and connected to both the lower control arm and upper control arm. Their assembly is completed in such a manner that the vibrations transmitted from them will gets absorbed by the suspension, that they're directly in touch with the tires. Hence there's a high chance of failure due to the sudden application of load and because of the formation of resonance. Their weight is high hence there's chance of generation of inertia forces and unnecessary material usage.

\section{Objectives}

- Modeling of existing Steering Upright using CATIA V5 R20 software.

- Analyzing for stresses and deformation of original Steering Upright using ANSYS Software. 
- Topological optimization for the Steering Upright for weight reduction.

- Performing Static and dynamic analysis over component to determine von misses stress and deformation.

- Perform the Harmonic and modal analysis of steering upright will be performed on ANSYS 19 software to find out the stress and deformation using Universal Testing Machine.

- Validation of the Experimental testing and correlating results.

\section{Methodology}

Step1: Literature survey and Component finalization.

Step2:3D Model drafting with the help of CATIA V5.

Step3: Modal \& Harmonic analysis of component by ANSYS uses FEA.

Step4: The Experimental Testing will be carried out.

Step5:Comparative analysis between the experimental \& analysis result to draw the final conclusion.

\section{Design \& analysis}

Three dimensional model of existing wheel hub is designed in Catia V5 R20 software.

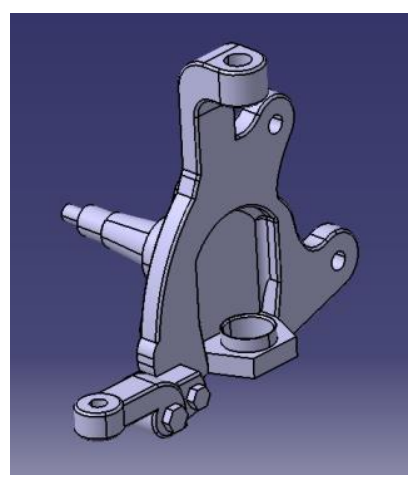

Figure 2: CATIA model of steering upright

\section{Calculations}

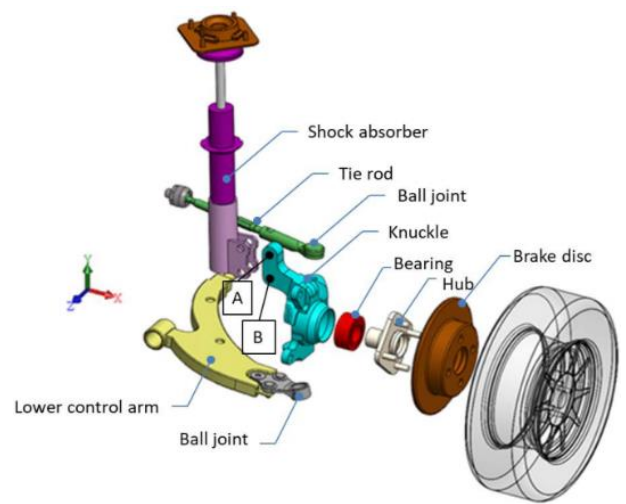

Figure 4: Different components assembly with steering upright

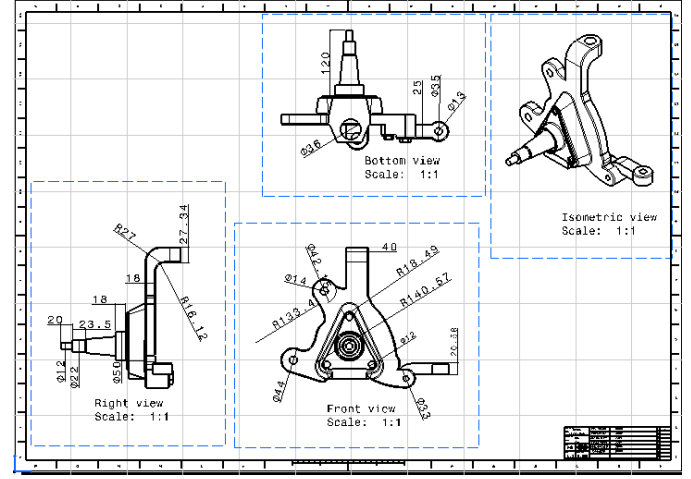

Figure 3: Drafting of Steering Upright

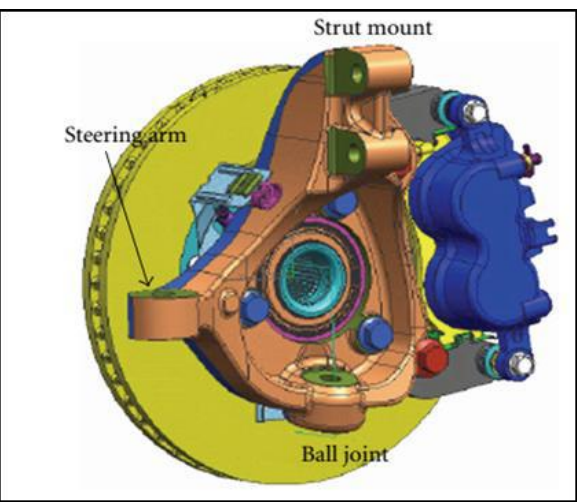

Figure 5: Assembly of steering 


\subsection{Overall Weight of Vehicle}

Kerb weight - vehicle weight when it is not loaded but full fuel tank is considered.

Gross weight - kerb weight + passengers + luggage

- Gross weight - $2625 \mathrm{~kg}$,

- Kerb weight $-1940 \mathrm{~kg}$,

- Braking force - $1.5 \mathrm{G}$,

- Lateral force $-1.5 \mathrm{G}$,

- Total weight of vehicle in Newton $=2625 \times 10=26250 \mathrm{~N}\left(\mathrm{~g}=10 \mathrm{~m} / \mathrm{s}^{2}\right)$,

- Weight on each wheel $=26250 / 4=6562.5 \mathrm{~N}$

(Brake moment of brake caliper applied during the motion),

- Diameter of disc brake - $0.250 \mathrm{~m}(250 \mathrm{~mm})$,

So, brake moment $=$ Force acting on each wheel $\mathrm{x}$ Perpendicular distance (radius of disc brake),

Moment $=6562.5 \times(250 / 2)=820312.5$ N.mm,

- Force on lower control arm is calculated by load acting on each wheel divided by 2 as it consists upper and lower control arm so load is distributed on each arm=6562.5 $/ 2=3281.5$ $\mathrm{N}$,

- Steering force applied by steering tie rod is generally considered as $100 \mathrm{~N}$ (it is maximum) according to papers as it is transferred through linking mechanism with steering.

- Force of strut is calculated by force acting on each wheel is $6562.5 \mathrm{~N}$ but strut is attached with spring which absorbs shock and load acting on strut so to function as a damper. So, nearly $40 \%$ of load is absorbed by spring due to which $=0.4 \times 6562.5=2625 \mathrm{~N}$.

These all forces are calculated as per literature papers mentioned in research papers and also for extreme or maximum conditions.

\subsection{FEA analysis in ANSYS}

\subsubsection{Material Selection - Structural Steel}

Table 1: Material properties of Structural Steel

\begin{tabular}{|c|c|c|c|}
\hline \multicolumn{4}{|c|}{ Properties of Outine Row 4: Structural Steel } \\
\hline & A & B & c \\
\hline 1 & Property & Value & Unit \\
\hline 2 & 7 Material Field Variables & Table & \\
\hline 3 & 7 Density & 7850 & $\mathrm{~kg} \mathrm{~m}^{\wedge}-3$ \\
\hline 4 & 曰跑 Isotropic Secant Coefficient of Thermal Expansion & & \\
\hline 5 & 7 Coefficient of Thermal Expansion & $1.2 \mathbb{E}-05$ & $C^{\wedge}-1$ \\
\hline 6 & 曰 Isotropic Elasticity & & \\
\hline 7 & Derive from & Young's Modu... & \\
\hline 8 & Young's Modulus & $2 E+11$ & $\mathrm{~Pa}$ \\
\hline 9 & Poisson's Ratio & 0.3 & \\
\hline 10 & Bulk Modulus & $1.6667 E+11$ & $\mathrm{~Pa}$ \\
\hline 11 & Shear Modulus & $7.6923 E+10$ & $\mathrm{~Pa}$ \\
\hline
\end{tabular}

\subsubsection{Mesh}

ANSYS Meshing may be a general-purpose, intelligent, automated high-performance product. It produces the foremost appropriate mesh for accurate, efficient Multi physics solutions. A mesh compatible for a selected analysis are often generated with one click for all parts during a model. Full controls over the choices wont to generate the mesh are available for the expert user who 
wants to fine-tune it. the facility of multiprocessing is automatically wont to reduce the time you've got to attend for mesh generation.

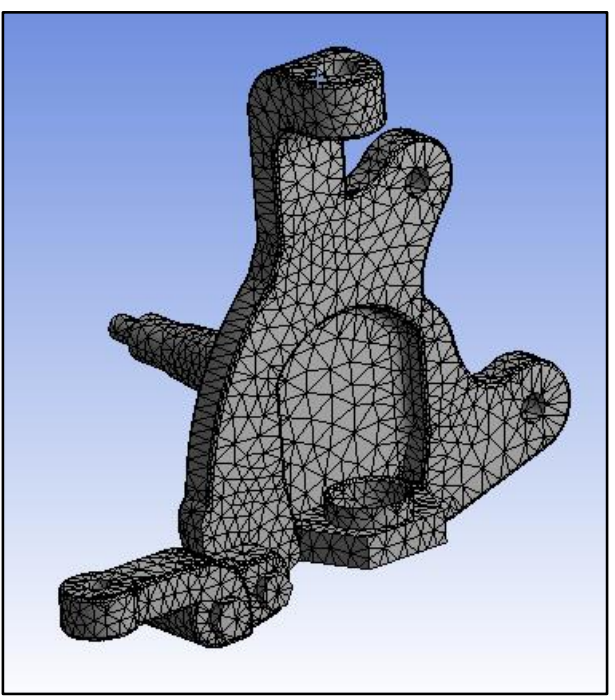

\begin{tabular}{|l|l|}
\hline Properties \\
\hline$\square$ Volume & $8.9725 \mathrm{e}+005 \mathrm{~mm}^{3}$ \\
\hline$\square$ Mass & $7.0434 \mathrm{~kg}$ \\
\hline
\end{tabular}

\begin{tabular}{|l|l|}
\hline Statistics \\
\hline$\square$ Nodes & 33489 \\
\hline$\square$ Elements & 18566 \\
\hline
\end{tabular}

Figure 6: Meshing of steering upright

After meshing of steering upright nodes are 33489 and elements 18566.

\subsubsection{Boundary Condition}

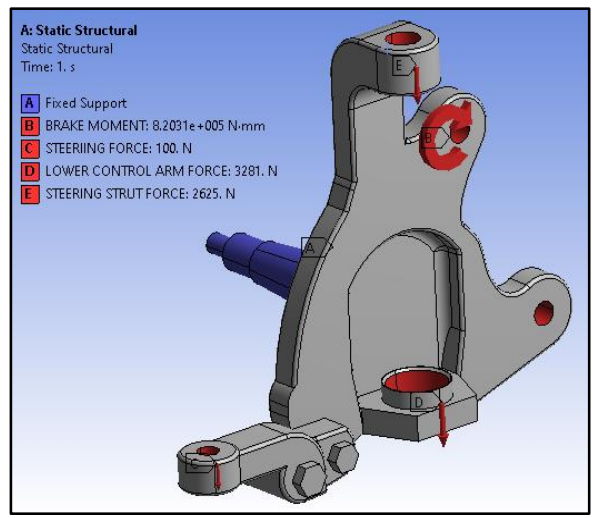

Figure 7:Details of boundary conditions for static analysis

- Boundary condition for steering upright is calculated analytically in calculation section and applied as per mentioned.

- Fixed support is applied at shaft behind the geometry indicated in blue Color as it is mounted in disc brake assembly.

- Brake moment is applied at two holes at right section it contains brake caliper with disc brake attachment with it. So, analytically moment is calculated and applied around it.

- Strut force is applied on upper surface with force in downward direction along with steering force on steering tie rod.

- In bottom lower control arm attachment is attached so force acting on lower control arm is calculated and applied on it. 


\subsection{Results}

\subsubsection{Total deformation}

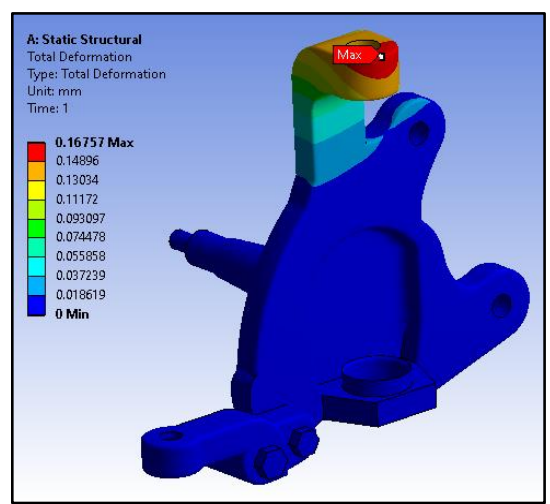

Figure 8:Total deformation results

- Maximum deformation under static condition of steering upright $0.167 \mathrm{~mm}$ is observed.

\subsubsection{Equivalent stress}

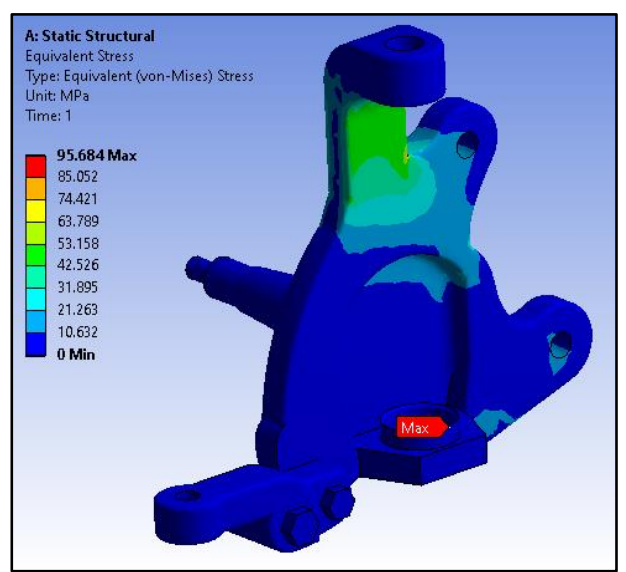

Figure 9: Equivalent stressof wheel hub

Maximum stress is observed around $95 \mathrm{MPa}$ at lower cross section (bottom indicated as MAX) but average stress induced is about $44 \mathrm{MPa}$ in green colour. So, design is safe compared to yield strength of material.

\subsection{Topology optimization}

Topology optimization may be a mathematical approach that optimizes material layout within a given design area, for a given set of loads and boundary conditions such that the resulting layout meets a prescribed set of performance targets.

\subsubsection{Basic Theory}

There are three kinds of structure optimization,

- Size optimization

- shape optimization 
- Topology optimization

Three optimization ways that correspond to the three stages of the product design methodology, significantly the detailed design, basic design and conceptual design. Size optimization keeps the structural form and topology structure invariant, to optimize the various parameters of structure, like thickness, section size of beam, materials properties; shape optimization maintains the topology structure, to vary the boundary of structure and form, search for the foremost applicable structure boundary scenario and shape; topology optimization is to hunt out the most effective path of materials distribution throughout never-ending domain that meet the displacement and stress conditions in structure, produce a selected performance optimum. Thus, compared to size and shape optimization, topology optimization with more freedom degree and larger design area, its greatest feature is below unsure structural form, in keeping with the well-known condition and a given load to figure out the cheap structure, every for the abstract variety of recent product and improvement design for existing product, it's the foremost promising side of structural optimization. For continuous structure topology optimization, there are some mature ways like: uniform technique, evolutionary structural optimization technique, variable density technique etc. Uniform technique introduced cell structure of micro structure (unit cell) at intervals the elements of the structure, each unit has three forms, significantly non-material voids $($ size $=1$ ), isotropic-material entity medium (size = 0 ) and orthotropic-material opening-hole medium $(0<$ size $<1)$. whereby the distribution of each form are able to describe the form of topology and conjointly the form of structure; evolutionary structural optimization technique believe that stress in any elements of the structure should beneath the identical level in an ideal structure. which suggests the native material with a low stress state isn't entirely used, thus you'll be able to delete the material artificially. Thus bit by bit remove material that in a low stress state, then delete the update rate, thus optimized structure becomes more uniform. Variable density technique is used to conduct optimization throughout this paper.

\subsubsection{Boundary Conditions for Topology Optimization}

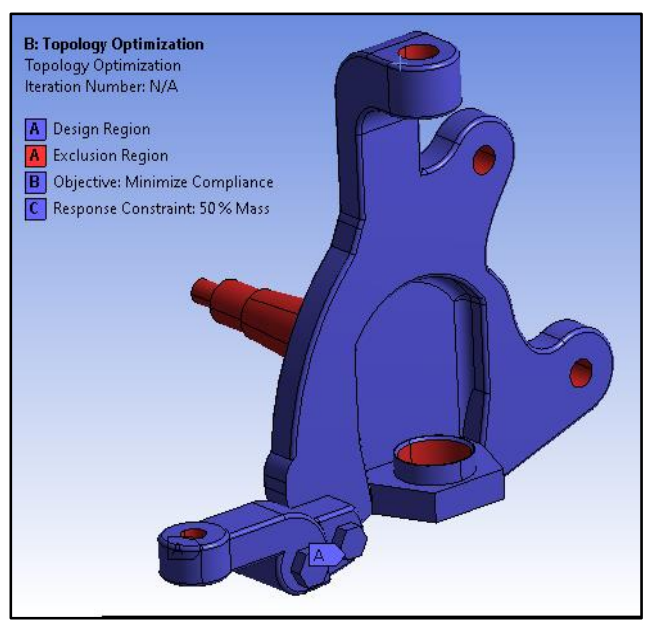

Figure 10: Details of boundary condition for topology optimization

In boundary condition non design area indicated in red region include the boundary condition in static structural analysis and design area is indicated in blue region. 

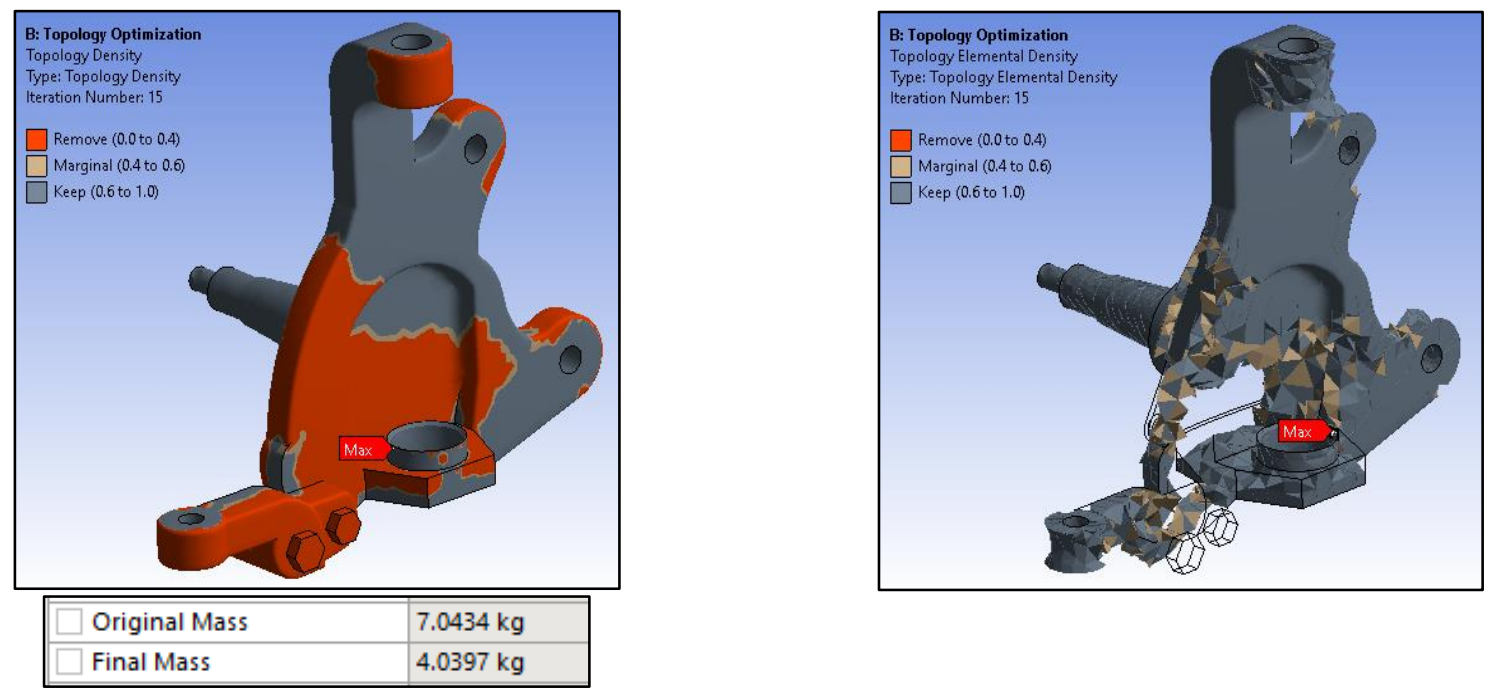

Figure 11: Topology optimization results

- Topology optimization is performed after static structural analysis with existing boundary condition to determine material removal area.

- After performing topology optimization red region indicates the material removal area from which material can be removed as per our need.

- So, in our case original mass is $7.04 \mathrm{~kg}$ but removal of material is about $55 \%$ which lead to $4.03 \mathrm{~kg}$ as per software. But it depends on us to removal of material by proper design and reanalysis as per existing conditions to sustain boundary condition.

\subsection{Excremental setup}

A universal testing machine (UTM), also called a universal tester, materials testing machine or materials test frame, is employed to check the strength and compressive strength of materials. An earlier name for a tensile testing machine may be a tensometer. The "universal" a part of the name reflects that it can perform many standard tensile and compression tests on materials, components, and structures (in other words, that it's versatile). The set-up and usage are detailed during a test method, often published by a standards organization. This defines the sample preparation, fixture, gauge length (length under observation), analysis, etc.

The specimen is placed within the machine between the grips and an extensometer if required can automatically record the change in gauge length during the test. If an extensometer isn't fitted, the machine itself can record the displacement between its cross heads on which the specimen is held. However, this method not only records the change long of the specimen but also all other extending / elastic components of the testing machine and its drive systems including any slipping of the specimen within the grips.

Once the machine is started it begins to use an increasing load on specimen. Throughout the tests the system and its associated software record the load and extension or compression of the specimen. 
Table 2: Specification of UTM

\begin{tabular}{|c|l|l|}
\hline 1 & Max Capacity & $400 \mathrm{KN}$ \\
\hline 2 & Measuring range & $0-400 \mathrm{KN}$ \\
\hline 3 & Least Count & $0.04 \mathrm{KN}$ \\
\hline 4 & Clearance for Tensile Test & $50-700 \mathrm{~mm}$ \\
\hline 5 & Clearance for Compression Test & $0-700 \mathrm{~mm}$ \\
\hline 6 & Clearance Between column & $500 \mathrm{~mm}$ \\
\hline 7 & Ram stroke & $200 \mathrm{~mm}$ \\
\hline 8 & Power supply & 3 Phase, 440 Volts, 50 Cycle. AC \\
\hline 9 & $\begin{array}{l}\text { Overall dimension of machine } \\
(\mathrm{L} * \mathrm{~W} * \mathrm{H})\end{array}$ & $2100 * 800 * 2060$ \\
\hline 10 & Weight & $2300 \mathrm{Kg}$ \\
\hline
\end{tabular}

\section{Conclusion}

- Modal analysis of steering upright is performed to obtain different mode shapes and natural frequency of existing steering upright and also it is observed that maximum frequency is around $2430 \mathrm{~Hz}$.

- Static structural analysis of steering upright is performed to determine deformation and equivalent stress. It is observed that around maximum deformation is $0.167 \mathrm{~mm}$ and equivalent stress is $95.7 \mathrm{MPa}$. An optimized model is obtained from topology optimization technique.

- It is concluded that the region indicated in red region in topology optimization provides information regarding removal of material from that area. In our case original mass is $7.04 \mathrm{~kg}$ but removal of material is to $4.03 \mathrm{~kg}$ as per software. But it depends on us to removal of material by proper design and reanalysis as per existing conditions to sustain boundary condition.

\section{References}

[1] Mahesh P. Sharma, Denish S. Mevawala, Harsh Joshi, Devendra A. Patel, Static Analysis of Steering Knuckle and Its Shape Optimization, e-ISSN: 2278-1684, p-ISSN: 2320-334X PP 34-38.

[2] Mr. V.Sivananth, Dr. S.Vijayarangan, Mr. R.Aswathaman , Fatigue and Impact Analysis of Automotive Steering Knuckle under Operating Load Cases.

[3] Gaurav Saxena, Ankit Singh Chauhan, Rohit Jain, Ishwar Gupta, Simulation and Optimization of wheel Hub and Upright of Vehicle: A Review, e-ISSN: 2278-1684,p-ISSN: 2320-334X, Volume 14, Issue 1 Ver. III (Jan. - Feb. 2017), PP 42-50 .

[4] Saksham Bhardwaj, Ashok B, Uma Shankar Lath, and Aman Agarwal, Design and Optimization of Steering Upright to Reduce the Weight Using FEA; SAE Technical Paper 2018-28-0081, 2018, doi:10.4271/2018-28-0081.

[5] Mahendra L. Shelar, Prof. H. P. Khairnar, Design Analysis and Optimization of Steering Knuckle Using Numerical Methods and Design of Experiments, 2014 IJEDR | Volume 2, Issue 3 | ISSN: 2321-9939.

[6] Razak, Ih.a., Yusop, M.Y.M., Yusop, M.S.M., Hashim, M.f, Modeling, simulation and optimization analysis of steering knuckle component for race car, IJRET eISSN: 2319-1163 | pISSN: 2321-7308.

[7] M Azmeer1, M H Basha, M F Hamid, M T A Rahman and M S M Hashim, "Design optimization of rear uprights for UniMAP Automotive Racing Team Formula SAE racing car", IOP Conf. Series: Journal of Physics: Conf. Series 908 (2017) 012051 doi :10.1088/1742-6596/908/1/012051. 
[8] S V Dusane, M K Dipke and M AKumbhalkar,Analysis of Steering Knuckle of All Terrain Vehicles (ATV) Using Finite Element Analysis, IOP Conf. Series: Materials Science and Engineering 149 (2016) 012133 doi:10.1088/1757-899X/149/1/012133.

[9] Fan Pingqing, Zhao Bo, Qiu Long,The Analysis on Destruction Forms of Steering Knuckle, 2011 Third International Conference on Measuring Technology and Mechatronics Automation.

[10] Mehrdad Zoroufi and Ali Fatemi, Fatigue Life Comparisons of Competing Manufacturing Processes: A Study of Steering Knuckle, SAE International by Univ of California Berkeley, Saturday, July 28, 2018. 